\title{
Human Chorionic Gonadotropin (hCG) Regression Curve for Predicting Response to EMA/CO (Etoposide, Methotrexate, Actinomycin D, Cyclophosphamide and Vincristine) Regimen in Gestational Trophoblastic Neoplasia
}

\author{
Athithan Rattanaburi*, Sathana Boonyapipat, Yuthasak Supasinth
}

\begin{abstract}
Background: An hCG regression curve has been used to predict the natural history and response to chemotherapy in gestational trophoblastic disease. We constructed hCG regression curves in high-risk gestational trophoblastic neoplasia (GTN) treated with EMA/CO and identified an optimal hCG level to detect EMA/CO resistance in GTN. Materials and Methods: Eighty-one women with GTN treated with EMA/CO were classified as primary high-risk GTN $(n=65)$ and single agent-resistance GTN $(n=16)$. The hCG levels prior to each course of chemotherapy were plotted in the 10th, 50th, and 90th percentiles to construct the hCG regression curves. Diagnostic performance was evaluated for an optimal cut-off value. Results: The median hCG levels were 264,482 $\mathrm{mIU} / \mathrm{mL} \mathrm{mIU} / \mathrm{mL}$ and $495.5 \mathrm{mIU} / \mathrm{mL} \mathrm{mIU} / \mathrm{mL}$ for primary high-risk GTN and single agent-resistance GTN, respectively. The 50th percentile of the hCG level in primary high-risk GTN and single agent-resistance turned to normal before the 4th and the 2nd course of chemotherapy, respectively. The 90th percentile of the hCG level in primary high-risk GTN and single agent-resistance turned to normal before the 9th and the 2nd course of chemotherapy, respectively. The hCG level of $\geq 118.6 \mathrm{mIU} / \mathrm{mL} \mathrm{mIU} / \mathrm{mL}$ at the 5 thcourse of EMA/CO predicted the EMA/CO resistance in primary high-risk GTN patients with a sensitivity of $85.7 \%$ and a specificity of $100 \%$. Conclusion: EMA/CO resistance in primary high-risk GTN can be predicted by using an hCG regression curve in combination with the cut-off value of $118.6 \mathrm{mIU} / \mathrm{mL}$ at the 5 thcourse of chemotherapy.
\end{abstract}

Keywords: EMA/CO - gestational trophoblastic neoplasia - human chorionic gonadotropin - drug resistance

Asian Pac J Cancer Prev, 16 (12), 5037-5041

\section{Introduction}

Gestational trophoblastic neoplasia (GTN) is a group of malignant lesions that arise from abnormal proliferation of placental trophoblast cells leading to invasive mole, choriocarcinoma, placental site trophoblastic tumor (PSTT), and epithelioid trophoblastic tumor (ETT). GTN is highly sensitive to chemotherapy with cure rate higher than $90 \%$ (Lurain, 2011)..

The patients with GTN are classified as having lowrisk or high-risk disease using the modified World Health Organization prognostic scoring system as adapted by the International Federation of Gynecology and Obstetrics (Ngan et al., 2003). High-risk is defined by FIGO stage IV or any FIGO stage with a score $\geq 7$ (Ngan et al., 2003), which indicates multi-agent chemotherapies for treatment. EMA/CO is an effective, less toxic regimen and the most frequently used primary therapy for high-risk GTN (Ngan et al., 2012).The remission rates of EMA/CO range from $71 \%$ to $86 \%$ (Bolis et al., 1988; Newlands et al., 1991; Bower et al., 1997; Escobar et al., 2003). The patients who were chemo-refractory to EMA/CO had a worse outcome compared to patients with relapsed disease (5-year survival 43\%) (Powles et al., 2007). Nowadays, there is no consensus guideline to define the criteria for resistance to chemotherapy even though some clinicians determine this condition by a plateau or a rising in serum human chorionic gonadotropin (hCG) and/or development of new metastases (van Trommel et al., 2006)..

To avoid the risk of ineffective cycles of chemotherapy, many studies attempted to develop new methods to enable early identification of chemoresistance in low-risk GTN such as an hCG regression nomogram (van Trommel et al., 2006) and serum hCG concentration ratios (van Trommel et al., 2008). Unfortunately, there is a lack of studies in patients with high-risk GTN.

In 2012, Lybol introduced the hCG regression nomogram in high-risk GTN to predict EMA/CO resistance. However, due to the limitations of diagnostic performance evidence, this nomogram could not be applied in clinical practice. Therefore, we wanted to study the pattern of hCG regression in high-risk GTN patients

Division of Gynecologic Oncology, Department of Obstetrics and Gynecology Faculty of Medicine, Prince of Songkla University, Songkhla, Thailand *For correspondence: $r$ _athithan123@hotmail.com 
Athithan Rattanaburi et al

and evaluate the diagnostic accuracy in predicting the risk of chemoresistance and assign a cut-off value for clinical application.

\section{Materials and Methods}

Following approval by the Research Ethics Committee, the medical records of patients with high-risk GTN who received EMA/CO chemotherapy regimen between 1 January 2002 and 31 December 2013 were reviewed. The patients who received EMA/CO for recurrent disease were excluded. The patients who were diagnosed as placental site trophoblastic tumor (PSTT) and epithelial trophoblastic tumor (ETT) were kept out. In addition, the patients whose serum hCG levels during EMA/CO treatment were unavailable were rejected from the study. A total of 94 patients treated with EMA/CO were included. Patient characteristics were reviewed for age, antecedent pregnancy, interval months from index pregnancy, size and site of metastasis, pretreatment serum hCG level, and serum hCG level prior to each course of chemotherapy. FIGO stage and FIGO risk prognostic scores were also recalculated to ensure precise data.

Based on the indication for treatment with EMA/CO, we classified the patients into either the primary high-risk GTN group $(n=77)$ or single agent-resistant group $(n=17)$.
Only the patients who reached complete remission after EMA/CO treatment in each group ( $n=65$ in the primary high-risk GTN, n=16 in the single agent resistance) were used separately to create the hCG regression curve. To approximate the normal distributions, the regression was based on $10 \log$ transformed serum hCG level for each chemotherapy course. The percentiles of $10 \%$ (P10), 50\% (P50), and 90\% (P90) were calculated and plotted for each course in an hCG regression curve using the program $\mathrm{R}$, version 2.14.2. The serum hCG level in each chemotherapy course of the patients who were resistant to EMA/CO were used to make receiver operating characteristic (ROC) curves and to calculate the area under the curves (AUCs). Chemoresistance was defined as at least 4 values of persistently elevated hCG plateau or sequential rise of hCG in 3 values in 2 weeks (van Trommel et al., 2006). The statistical analyses were performed using the $\mathrm{R}$ program, version 2.14.2. Differences in the numerical data between the two groups were tested nonparametrically (Mann-Whitney U test) and parametrically (Student's T test). All tests were considered significantly different at $\mathrm{P}<0.05$.

\section{Immunoassays}

All hCG concentrations were measured using electrochemiluminescence immunoassay (ECLIA) with

\section{Table 1. Patient Characteristics}

\begin{tabular}{|c|c|c|c|}
\hline & $\begin{array}{c}\text { Primary high-risk } \\
n=65\end{array}$ & $\begin{array}{l}\text { Single agent resistance } \\
\mathrm{n}=16\end{array}$ & $\mathrm{p}$ value \\
\hline Age (years) & 39 & 39.5 & 0.79 \\
\hline Median (Min-Max) & $(17-57)$ & $(21-51)$ & \\
\hline Parity (Median) (Min-Max) & 2 & 2 & 0.1 \\
\hline Antecedent pregnancy & $(0-10)$ & $(0-6)$ & 0.02 \\
\hline Hydatidiform mole (\%) & $27(41.5 \%)$ & $13(81.2 \%)$ & \\
\hline Non-molar abortion (\%) & $16(24.6 \%)$ & $1(6.2 \%)$ & \\
\hline Term pregnancy $(\%)$ & $22(33.8 \%)$ & $2(12.5 \%)$ & \\
\hline Interval from index & 11 & 9.5 & 0.74 \\
\hline pregnancy (months) & $(1-312)$ & $(2-168)$ & \\
\hline$<4$ & $14(21.5 \%)$ & $3(18.8 \%)$ & \\
\hline $4-6$ & $11(16.9 \%)$ & $3(18.8 \%)$ & \\
\hline $7-12$ & $9(13.8 \%)$ & $4(25.0 \%)$ & \\
\hline$>12$ & $31(47.7 \%)$ & $6(37.5 \%)$ & \\
\hline Pretreatment hCG (mIU/mL) (Min-Max) & 264482 & 495.5 & $<0.001$ \\
\hline Median (Min-Max) & $(3173-4000000)$ & $(70-34400)$ & \\
\hline Tumor size (cm.) & 6 & 2 & $<0.001$ \\
\hline Median (Min-Max) & $(0-16)$ & $(0-7)$ & \\
\hline Metastasis disease & $45(69.2 \%)$ & $3(18.8 \%)$ & $<0.001$ \\
\hline Vagina & $14(21.5 \%)$ & 0 & \\
\hline Lung & $37(56.9 \%)$ & $2(12.5 \%)$ & \\
\hline Spleen, Kidney & $1(1.5 \%)$ & 0 & \\
\hline GI & $2(3.1 \%)$ & 0 & \\
\hline Liver, Brain & $11(16.9 \%)$ & $1(6.2 \%)$ & \\
\hline FIGO stage & & & 0.006 \\
\hline I & $21(32.3 \%)$ & $13(81.2 \%)$ & \\
\hline II & $5(7.7 \%)$ & 0 & \\
\hline III & $29(44.6 \%)$ & $2(12.5 \%)$ & \\
\hline IV & $10(15.4 \%)$ & $1(6.2 \%)$ & \\
\hline FIGO score & 12 & 8 & $<0.001$ \\
\hline Median (Min-Max) & $(7-21)$ & $(1-11)$ & \\
\hline No. of chemotherapy & $6(3-11)$ & $3(2.5)$ & $<0.001$ \\
\hline Median (Min-Max) & (3-11) & $(2-5)$ & \\
\hline
\end{tabular}


HCG Regression Curve for Predicting Response to EMA/CO Regimen in High-risk Gestational Trophoblastic Neoplasia

the Elecsys 1010/2010, Modular Analytics E170, cobas e601/411 (Roche diagnostics). This assay determined intact hCG plus the hCG B-subunit in human serum and plasma. This method was standardized against the $4^{\text {th }}$ International Standard for Chorionic Gonadotropin from the National Institute for Biological Standards and Control (NIBSC) code 75/589. Serum hCG concentrations were considered to be normal if less than $7 \mathrm{mIU} / \mathrm{mL}$. The corresponding $95 \%$ confidence interval extends to an upper limit of $8.3 \mathrm{mIU} / \mathrm{mL}$. In the author's institute, hCG levels less than $10 \mathrm{mIU} / \mathrm{mL}$ were considered normal.

\section{Results}

The characteristics of patients with primary high-risk GTN ( $n=65)$ and patients with single agent-resistance $(n=16)$ are shown in Table 1. Expectedly, the median pretreatment serum hCG levels of primary high-risk GTN patients were significantly elevated compared to those with single agent -resistance (264,482 vs. $495.5 \mathrm{mIU} / \mathrm{mL}$, $\mathrm{P}<0.001)$. The median tumor size also was significantly larger in the primary high-risk GTN group compared to the single agent-resistant patients ( 6 vs. $2 \mathrm{~cm}, \mathrm{P}<0.001$ ). More metastatic diseases was observed in the primary high-risk GTN group (69.2\% vs. $18.8 \%, \mathrm{P}<0.001)$. The

Table 2. Diagnostic Performance of Each Course of Chemotherapy

\begin{tabular}{lccc}
\hline Course & $3^{\text {rd }}$ & $4^{\text {th }}$ & $5^{\text {th }}$ \\
\hline Study, No of patients & 12 & 8 & 7 \\
Control, No of patients & 77 & 70 & 61 \\
hCG cut-off (mIU/mL) & $\geq 55$ & $\geq 118.3$ & $\geq 118.6$ \\
Sensitivity & $100 \%$ & $87.50 \%$ & $85.70 \%$ \\
Specificity & $78.40 \%$ & $95.10 \%$ & $100 \%$ \\
AUC & 0.892 & 0.913 & 0.928 \\
\hline *Abbreviation: AUC, area under the curve & &
\end{tabular}
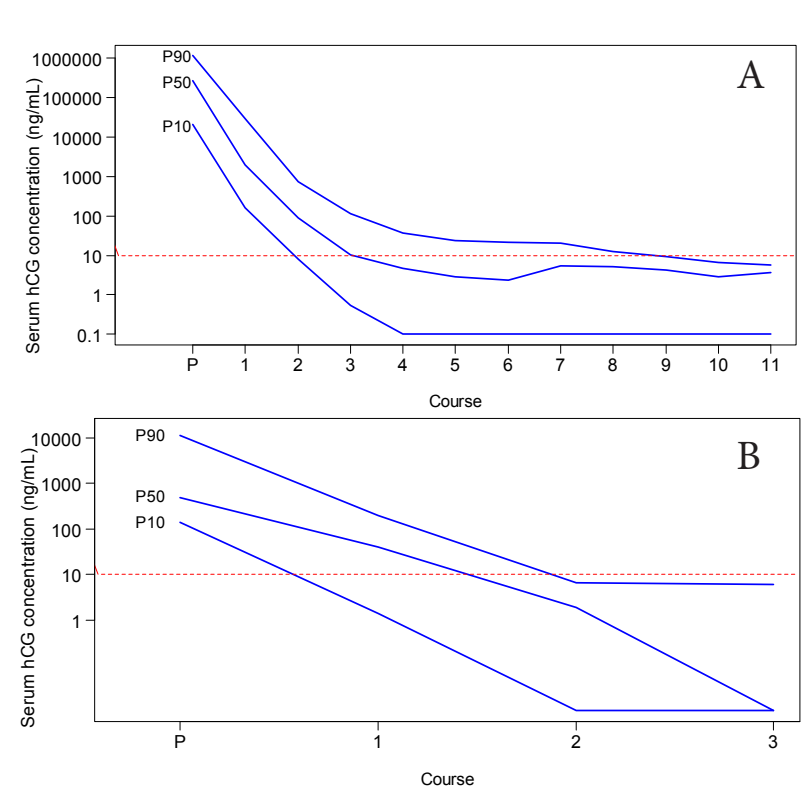

Figure 1. A) Normal hCG Regression Curve in the Patients who Successfully Treated with EMA/CO for Primary High Risk GTN $(n=65)$; B) Normal hCG Regression Curve in the Patients who Successfully Treated with EMA/CO Single Agent-Resistance (n=16) lungs were the most common site of metastasis in both groups. No differences in age, parity, and the number of interval months from index pregnancy were observed between the 2 groups.

Not surprisingly, the FIGO stage for the primary high-risk GTN group was mostly stage III while the FIGO stage for the single agent-resistant patients was mostly stage I. The median FIGO prognostic score of primary high-risk GTN patients was also significantly higher than the patients with single agent-resistance (12 vs. $8, \mathrm{P}<0.001)$. There was a greater number of EMA/ $\mathrm{CO}$ courses administered to the primary high-risk GTN patients (median 6 courses, range 3-11) compared with the single agent-resistant patients (median 3 courses, range $2-5, \mathrm{P}<0.001)$.

The hCG regression curve for the patients who were successfully treated with EMA/CO for primary highrisk GTN is shown in Figure $1 \mathrm{~A}$. The $90^{\text {th }}$ percentile of the pretreatment hCG levels was $1,186,653 \mathrm{mIU} / \mathrm{mL}$ and turned to a normal level before the 9th course of chemotherapy. The median pretreatment hCG level of this group was $264,482 \mathrm{mIU} / \mathrm{mL}$. Half of the patients with primary high-risk GTN had a normal hCG level before the start of the $4^{\text {th }}$ course of chemotherapy. The $10^{\text {th }}$ percentile of pretreatment hCG level of this group was 20,912.6 mIU/ $\mathrm{mL}$ and turned to a normal level before the $2^{\text {nd }}$ course of chemotherapy.

The hCG regression curve for the patients with single agent-resistance who were successfully treated with EMA/ $\mathrm{CO}$ is shown in Figure 1. B. The 90th percentile of the pretreatment hCG level was $11,419.5 \mathrm{mIU} / \mathrm{mL}$. Ninety percent of the patients with single agent-resistance had normal hCG levels before the 2 nd course of chemotherapy. The median pretreatment hCG level of this group was $495.5 \mathrm{mIU} / \mathrm{mL}$ and half of the patients had a normal hCG level before the $2^{\text {nd }}$ course of chemotherapy. The 10th percentile of pretreatment hCG level was $136.5 \mathrm{mIU} / \mathrm{mL}$.

The hCG levels before the start of each chemotherapy of the patients with primary high-risk GTN who were resistant to EMA/CO $(n=12)$ were plotted in an hCG regression curve as shown in Figure 2. The diagnostic accuracy to predict the patients who may be resistant to EMA/CO treatment in each course was performed by calculation of the AUC of an ROC curve in the 3rd, 4th, and 5thcourses of chemotherapy (Figures 3A-C). The corresponding AUCs for these ROCs are listed in Table 2. The AUC of the hCG level before the start of

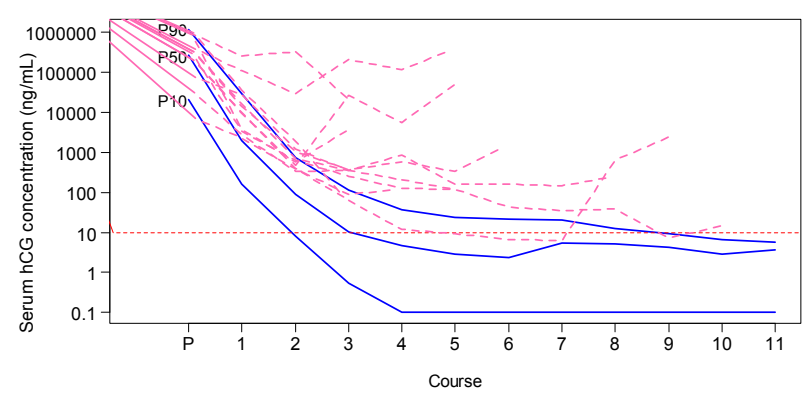

Figure 2. Serum hCG Regression of the Primary High Risk GTN with EMA/CO Resistance $(n=12)$ were Plotted in the hCG Regression Curve 

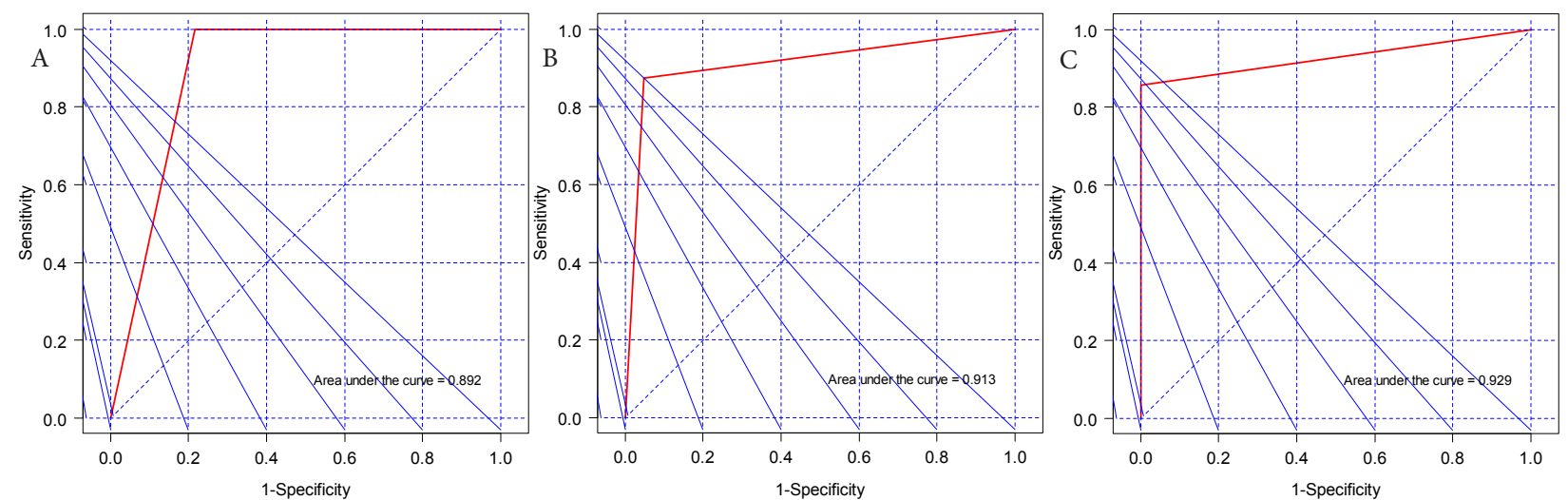

Figure 3. A) ROC Analysis at the Third Course of EMA/CO for the Patient with EMA/CO Resistance; B) ROC Analysis at the Forth Course of EMA/CO for the Patient with EMA/CO resistance; C) ROC Analysis at the Fifth Course of EMA/CO for the Patient with EMA/CO Resistance

the $3^{\text {rd }}$ EMA/CO treatment was 0.892. The AUC of the hCG level before the start the $4^{\text {th }} \mathrm{EMA} / \mathrm{CO}$ treatment was 0.913 , which increased to 0.928 preceding the $5^{\text {th }} \mathrm{EMA} /$ $\mathrm{CO}$ treatment. In accordance with the AUC, an hCG level $\geq 118.6 \mathrm{mIU} / \mathrm{mL}$ measured prior to the $5^{\text {th }}$ course of chemotherapy provided the best diagnostic accuracy in predicting EMA/CO regimen resistance (Sensitivity $85.7 \%$, specificity $100 \%$ ).

\section{Discussion}

The objectives of the study were to construct the hCG regression curves for the patients with high-risk GTN who were successfully treated with EMA/CO in different indications and use these curves to calculate the diagnostic performance and predict EMA/CO resistance. The median pretreatment hCG level in the primary high-risk GTN group was significantly higher than in the single agent-resistant group due to the larger tumor volumes. The $90^{\text {th }}$ percentile was chosen as the upper line of the hCG regression curve because of the concern for early prediction of EMA/CO resistance as was found in a previous study (Lybol et al., 2012). We found that half of the patients in primary high-risk GTN and single agent-resistance had normal hCG levels before the start of the $4^{\text {th }}$ and the $2^{\text {nd }}$ course of chemotherapy, respectively. Likewise, Lybol et al. (2012) reported half of the patients with primary high-risk GTN and MTX-resistance had normal hCG levels before the 6th and the $3^{\text {rd }}$ course of chemotherapy, respectively. For the $90^{\text {th }}$ percentile in our study, the patients with primary high-risk GTN had normal hCG levels before the $9^{\text {th }}$ course while the single agent-resistance patients had normal hCG levels before the $2^{\text {nd }}$ course of chemotherapy. Comparing our results with the Lybol et al. (2012) study, they reported that the 90th percentile of the hCG level turned to normal before the $8^{\text {th }}$ and the $4^{\text {th }}$ course in primary high-risk GTN and MTX resistant patients, respectively.

There is a particular concern for the exposure of ineffective courses of chemotherapy. Many studies tried to identify early and accurate methods in detection of chemoresistance in low-risk GTN patients (van Trommel et al., 2006). They found a $97.5 \%$ specificity of the hCG level in the first few courses of MTX that identified
$50 \%$ of the patients who were likely to need alternative chemotherapy to cure their disease. Additionally, Lybol et al. (2012) constructed an hCG regression nomogram for the high-risk GTN patients treated with EMA/CO. They did not explore the diagnostic accuracy of serum hCG level to detect EMA/CO resistance in high-risk GTN patients. To the best of our knowledge, this is the first study that explores the diagnostic performance of an $\mathrm{hCG}$ regression curve in patients with high-risk GTN treated with EMA/ $\mathrm{CO}$ to identify the EMA/CO resistance.

We selected the $3^{\text {rd }}, 4^{\text {th }}$, and 5 th course of chemotherapy to calculate the diagnostic performance of EMA/CO resistance. There were 2 reasons. First, this selection leads to a more precise early detection of EMA/CO resistance because most of the patients with EMA/CO resistance had hCG levels higher than the 90th percentile of the hCG regression curve after the $3^{\text {rd }}$ course of chemotherapy. If we had selected the $1^{\text {st }}$ or $2^{\text {nd }}$ course, it may have led to an over diagnosis. The second is to reduce the side effects from an ineffective course of chemotherapy. Consequently, we chose the $3^{\text {rd }}$ to $5^{\text {th }}$ courses to calculate the diagnostic accuracy. Of interest, we found the highest specificity $(100 \%)$ and a good sensitivity $(85.7 \%)$ to predict EMA/ $\mathrm{CO}$ resistance in primary high-risk GTN patients by using the hCG cut-off level of $\geq 118.6 \mathrm{mIU} / \mathrm{mL}$ at the $5^{\text {th }}$ course of EMA/CO chemotherapy. The use of this value would avoid an excessive treatment, from the absence of false positive result. Nevertheless, because of a lack of consensus diagnostic criteria for chemotherapy resistance and the application of rising or plateau criteria in some institutes, we suggest that if the serum hCG meets the criteria of chemoresistance before the $5^{\text {th }}$ course of EMA/ $\mathrm{CO}$, an alternative criteria of rising or plateau hCG level would be helpful.

In patients who show resistance to the EMA/CO regimen, second line chemotherapy maybe considered. Several effective regimens of salvage chemotherapy were proposed such as EP/EMA (Bower et al., 1997; Newlands et al., 2000), TP/TE (Wang et al., 2008), and Capecitabine (Bianconi et al., 2007). Some regimens were less effective in a salvage setting such as 5-fluouracil plus Actinomycin D (Manopunya and Suprasert, 2012), and VAC (Oranratanaphan and Lertkhachonsuk, 2014). In some circumstances, hysterectomy may be required 
HCG Regression Curve for Predicting Response to EMA/CO Regimen in High-risk Gestational Trophoblastic Neoplasia

when a chemoresistant lesion was confined to the uterus (Pongsaranantakul and Kietpeerakool, 2009). In this study, five of twelve patients with primary high-risk GTN who showed resistance to EMA/CO regimen had disease remission by salvage chemotherapy with EP/EMA regimen (3), hysterectomy (1), and hysterectomy followed by EP/EMA (1). Two patients were lost to follow-up during second line chemotherapy treatment. Regrettably, another five patients failed to achieve remission; 3 patients died with disease progression and 2 patients died with febrile neutropenia during chemotherapy treatment. A patient in the MTX resistant group who failed EMA/ CO treatment had disease remission with hysterectomy followed with the TP/TE regimen.

The limitation of this study is that the number of patients with single agent-resistance was too small to evaluate the diagnostic accuracy. Further prospective trials may consider the use of the hCG regression curve compared with rising or plateau criteria to study the survival and adverse effects of chemotherapy.

\section{References}

Bianconi M, Jankilevich G, Otero S, Nassif J, Storino C(2007). Successful salvage of a relapsed high risk gestational trophoblastic neoplasia patient using capecitabine. Gynecol Oncol, 106, 268-71.

Bolis G, BonazziC, Landoni F, et al (1988). EMA/CO regimen in high-risk gestational trophoblastic tumor (GTT). Gynecol Oncol, 31, 439-44.

Bower M, Newlands ES, Holden L, et al (1997). EMA/CO for high-risk gestational trophoblastic tumors: results from a cohort of 272 patients. J Clin Oncol, 15, 2636-43.

Escobar PF, Lurain JR, Singh DK, Bozorgi K, Fishman DA (2003). Treatment of high-risk gestational trophoblastic neoplasia with etoposide, methotrexate, actinomycin D, cyclophosphamide, and vincristine chemotherapy. Gynecol Oncol, 91, 552-7.

Lurain JR (2011). Gestational trophoblastic disease II: classification and management of gestational trophoblastic neoplasia. Am J Obstet Gynecol, 204, 11-8.

LybolC, Westerdijk K, Sweep FC, et al (2012). Human chorionic gonadotropin (hCG) regression nomograms for patients with high-risk gestational trophoblastic neoplasia treated with EMA/CO (etoposide, methotrexate, actinomycin D, cyclophosphamide and vincristine) chemotherapy. Ann Oncol, 23, 2903-06.

Manopunya M, Suprasert P (2012). Resistant gestational trophoblastic neoplasia patients treated with 5-fluouracil plus actinomycin D. Asian Pac J Cancer Prev, 13, 387-90.

Newlands ES, Bagshawe KD, Begent RH, Rustin GJ, Holden L(1991). Results with the EMA/CO (etoposide, methotrexate, actinomycin D, cyclophosphamide, vincristine) regimen in high risk gestational trophoblastic tumours, 1979 to 1989. Br J Obstet Gynaecol, 98, 550-7.

Ngan HY, Bender H, Benedet JL, et al (2003). Gestational trophoblastic neoplasia, FIGO 2000 staging and classification. Int J Gynaecol Obstet, 83, 175-7.

Ngan HYS, Kohorn EI, Cole LA, et al (2012). Trophoblastic disease. Int J Gynecol Obstet, 119, 130-6.

Oranratanaphan S, Lertkhachonsuk R (2014). Treatment of extremely high risk and resistant gestational trophoblastic neoplasia patients in king chulalongkorn memorial hospital. Asian Pac J Cancer Prev, 15, 925-8.

Pongsaranantakul S, Kietpeerakool C (2009). Hysterectomy in gestational trophoblastic neoplasia: Chiang Mai University Hospital's experience. Asian Pac J Cancer Prev, 10, 311-4.

Powles T, Savage PM, Stebbing J, et al (2007). A comparison of patients with relapsed and chemo-refractory gestational trophoblastic neoplasia. Br J Cancer, 96, 732-7.

Shigematsu T, Kamura T, Saito T, et al (1998). Identification of persistent trophoblastic diseases based on a human chorionic gonadotropin regression curve by means of a stepwise piecewise linear regression analysis after the evacuation of uneventful moles. Gynecol Oncol, 71, 376-80.

van Trommel NE, Massuger LF, Schijf CP, et al (2006). Early identification of resistance to first-line single-agent methotrexate in patients with persistent trophoblastic disease. J Clin Oncol, 24, 52-8.

van Trommel NE, Ngo Duc H, Massuger LF, et al (2008). Early identification of persistent trophoblastic disease with serum hCG concentration ratios. Int J Gynecol Cancer, 18, 318-23.

WangJ, Short D, Sebire NJ, et al (2008). Salvage chemotherapy of relapsed or high-risk gestational trophoblastic neoplasia (GTN) with paclitaxel/cisplatin alternating with paclitaxel/ etoposide (TP/TE). Ann Oncol, 19, 1578-83. 